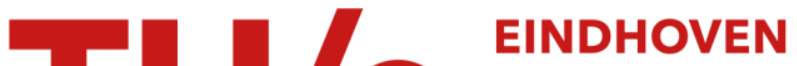 UNIVERSITY OF TECHNOLOGY
}

\section{A $1.25 \mu \mathrm{J}$ per Measurement Ultrasound Rangefinder System in 65nm CMOS for Explorations With a Swarm of Sensor Nodes}

\section{Citation for published version (APA):}

Berkol, G., Baltus, P. G. M., Harpe, P. J. A., \& Cantatore, E. (2021). A $1.25 \mu \mathrm{J}$ per Measurement Ultrasound Rangefinder System in 65nm CMOS for Explorations With a Swarm of Sensor Nodes. IEEE Transactions on Circuits and Systems I: Regular Papers, 68(4), 1409-1420. [9329156].

https://doi.org/10.1109/TCSI.2021.3049246

\section{Document license:}

TAVERNE

DOI:

10.1109/TCSI.2021.3049246

Document status and date:

Published: 01/04/2021

\section{Document Version:}

Publisher's PDF, also known as Version of Record (includes final page, issue and volume numbers)

\section{Please check the document version of this publication:}

- A submitted manuscript is the version of the article upon submission and before peer-review. There can be important differences between the submitted version and the official published version of record. People interested in the research are advised to contact the author for the final version of the publication, or visit the $\mathrm{DOI}$ to the publisher's website.

- The final author version and the galley proof are versions of the publication after peer review.

- The final published version features the final layout of the paper including the volume, issue and page numbers.

Link to publication

\footnotetext{
General rights

- You may freely distribute the URL identifying the publication in the public portal. follow below link for the End User Agreement:

www.tue.nl/taverne

\section{Take down policy}

If you believe that this document breaches copyright please contact us at:

openaccess@tue.nl

providing details and we will investigate your claim.
}

Copyright and moral rights for the publications made accessible in the public portal are retained by the authors and/or other copyright owners and it is a condition of accessing publications that users recognise and abide by the legal requirements associated with these rights.

- Users may download and print one copy of any publication from the public portal for the purpose of private study or research.

- You may not further distribute the material or use it for any profit-making activity or commercial gain

If the publication is distributed under the terms of Article 25fa of the Dutch Copyright Act, indicated by the "Taverne" license above, please 


\title{
A $1.25 \mu \mathrm{J}$ per Measurement Ultrasound Rangefinder System in $65 \mathrm{~nm}$ CMOS for Explorations With a Swarm of Sensor Nodes
}

\author{
Gönenç Berkol ${ }^{\circledR}$, Member, IEEE, Peter G. M. Baltus ${ }^{\circledR}$, Senior Member, IEEE, \\ Pieter J. A. Harpe $\left.{ }^{(}\right)$Senior Member, IEEE, and Eugenio Cantatore ${ }^{\circledR}$, Fellow, IEEE
}

\begin{abstract}
This paper presents an ultrasound rangefinder system able to find relative distances among energy-constrained sensor nodes. The nodes build a swarm that is operated in collision and multipath rich environments. A new distance measurement technique combining Wake-up and Frequency Modulated Continuous Wave (FMCW) is proposed to enable the ranging while neglecting the echoes from passive reflectors in the environment. The building blocks of the sensor nodes comprise a transmitter, a wake-up receiver, and a ranging receiver, all implemented in a $65 \mathrm{~nm}$ CMOS technology. The transmitter includes two switched-capacitor converters and an output multiplexer to generate a four-level driving signal and broadcast either a wake-up sequence or a digitally synthesized ultrasound Chirp. The transmitter dissipates $0.43 \mu \mathrm{J}$ and $0.82 \mu \mathrm{J}$ to broadcast the wake-up signal and the Chirp, respectively. A mixer first architecture is exploited in the wake-up receiver to reduce the always-on power consumption of the nodes. The ranging receiver uses a heterodyne architecture suited for the FMCW. The power consumption of the wake-up receiver and ranging receiver is 23.6 $\mathrm{nW}$ and $0.56 \mu \mathrm{W}$, respectively. The proposed rangefinder is experimentally characterized up to a $1 \mathrm{~m}$ distance in air and dissipates $1.25 \mu \mathrm{J}$ per measurement, achieving a resolution of $18.7 \mathrm{~mm}$ at $0.55 \mathrm{~m}$.
\end{abstract}

Index Terms-Ultrasound rangefinder, ultrasound front-end, ultrasound receiver, analog IC, swarm of sensor nodes.

\section{INTRODUCTION}

$\mathbf{U}$ LTRASOUND (US) has been used in emerging applications for the exploration of the ocean floor [1], underground infrastructures [2], water distribution systems, and industrial tanks for processing chemicals [3]-[7]. In these applications different fluidic environments are explored with a swarm of sensor nodes, to gather data on the shape and dimensions of enclosed volumes that are missing GPS coverage. Sensors should be autonomous as human intervention during the exploration is not possible. Miniaturized and energy-constrained sensor nodes are needed considering the limited size of these environments. US is preferred for low bit-rate and energy efficient data exchange and ranging

Manuscript received July 28, 2020; revised December 24, 2020; accepted December 31, 2020. Date of publication January 20, 2021; date of current version March 8, 2021. This work was supported by the European Union's Horizon 2020 Research and Innovation Programme under Grant 665347. This article was recommended by Associate Editor D. Zhao, (Corresponding author: Gönenç Berkol.)

The authors are with the Electrical Engineering Department, IC Group, Eindhoven University of Technology, 5600 MB Eindhoven, The Netherlands (e-mail: g.berkol@tue.nl).

Color versions of one or more figures in this article are available at https://doi.org/10.1109/TCSI.2021.3049246.

Digital Object Identifier 10.1109/TCSI.2021.3049246 between the nodes, as US propagates with a lower attenuation in fluids compared to RF [8]. The nodes are injected into the volume to explore and perform distance measurements among them while going with the flow of the medium [9]. Their mutual distance measurements are recorded to their memory as they traverse the volume. These data are analyzed after recuperating the sensor nodes. Data post-processing is performed at central computing stations [10], [11], to extract information about the geometry of the volumes explored by the swarm, based on the mutual distance measurements performed.

Most ranging methods proposed in literature [12] are based on either using additional hardware like GPS or having a beacon in the network that has the capability to communicate with the outside world and the other sensor nodes [13]. Both approaches are not suited for the applications considered in this paper. Moreover, the distance measurement methods that are based on Received Signal Strength Indicator (RSSI), and Time of Arrival (ToA) require a global network synchronization [14], together with highly stable timing sources (such as atomic clocks) and advanced MAC protocols [15] that require exchanging several data packets. These approaches are too power hungry for our applications. The Time Difference of Arrival (TDoA) based distance measurement methods take advantage of the different propagation speeds of US and RF in the operating medium. However, their maximum range is limited due to the attenuation of RF in fluids [8]. Typical US distance measurement methods exploit Time of Flight (ToF) of the US echoes coming from passive reflectors [16]-[18]. However, collisions and multipath propagation of the echoes experienced when the nodes are operated in an enclosed volume [19] increase the complexity of extracting distance information from the measurements and decrease the reliability when reconstructing the geometry of the explored volumes. As a result, a new distance measurement method able to cope with the echoes reflected from the boundary of the enclosed volumes while minimizing US data-exchange needed to support synchronization is required. To address the aforementioned challenges, a novel US rangefinder system based on a Wake-up and a Frequency Modulated Continuous Wave (FMCW) based ranging is proposed in this paper. An important feature of the proposed system is that the sensor nodes use a wake-up link, which provides ultra low power consumption, while the FMCW method is exploited in a ranging link to find the relative distance among the 


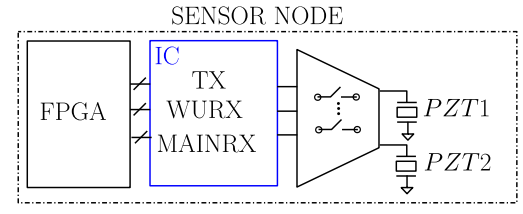

a)
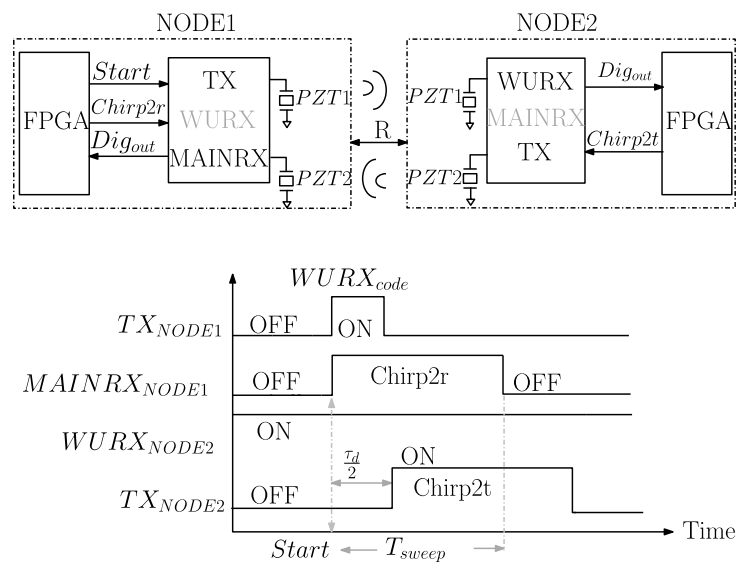

b)

Fig. 1. a) Building blocks of the sensor node. b) Illustration of the proposed ranging concept including two sensor nodes, NODE1 and NODE2, which are activating their corresponding hardware (not to scale).

nodes in the swarm. Furthermore, the wake-up and FMCW ranging links use separate frequency bands, therefore passive reflections from the boundary of the enclosed volume and active responses of the nodes can be easily distinguished. The paper is organized as follows: In Sections II and III, high-level system and circuit design are discussed. The detailed circuit implementation and the measurement results are presented in Section IV and Section V, respectively. The performance of the US rangefinder is benchmarked against prior art in Section VI and conclusions are provided in Section VII.

\section{US RANGEFINDER SYSTEM}

\section{A. System Operation}

The proposed rangefinder system is based on identical sensor nodes. Their block diagram is depicted in Fig. 1a, where each node consists of two off-chip piezoelectric transducers (PZT1 and PZT2); a transmitter (TX), a wake-up receiver (WURX), a ranging receiver (MAINRX) integrated on-chip, and a digital backend implemented on an FPGA. PZT1 and PZT2 are routed to corresponding hardware with an off-chip switching matrix. PZT1 and PZT2 have different resonance frequencies, thereby their operating frequency bands are separated. The nodes can be configured in two different modes of operation. The first one is called the initiator mode, where the node starts the ranging protocol to find its relative distance to the surrounding nodes. The second operating mode is called the wake-up mode. In this state, the sensor nodes listen to the environment via PZT1 and WURX. If the level of the incoming signal exceeds a pre-defined threshold, the nodes wake-up and transmit back a frequency-modulated response. The sensors operating in wake-up mode activate only their WURX, while TX and MAINRX are switched-off. Therefore, power consumption in this mode is extremely limited.
Fig. 1b demonstrates an example of the proposed rangefinder system, where the ranging between two sensor nodes, NODE1 and NODE2 at a distance R, together with the timing diagram illustrating the activation of the corresponding blocks in the nodes, are depicted. Here, NODE1 (in initiator mode) starts the ranging by broadcasting a $W U R X_{\text {code }}$ to the environment via PZT1. At the same time, NODE1 activates its MAINRX to listen to the responses from the nearby nodes. The activation of the initiator mode is controlled via a Start input generated by the digital backend without any need for global synchronization in the network. As illustrated in Fig. 1b, a digitally synthesized US signal, Chirp2r, is generated by the FPGA and used in NODE1 as a mixing signal in its MAINRX, which listens to PZT2. Chirp2r (Fig. 2) is a frequency-modulated US signal whose frequency increases linearly with time in the interval $0-T_{\text {sweep. }}$. Meanwhile, NODE2 is in the wake-up mode. The $W U R X_{\text {code }}$ reaches NODE2 after a propagation delay $\frac{\tau_{d}}{2}$, is received by its PZT1 and transformed to a digital representation via WURX. This is followed by a digital comparison performed in the FPGA of NODE2. If the digital output of WURX exceeds a pre-defined threshold, the TX in NODE2 is immediately activated to broadcast a response, Chirp2t, via PZT2. Chirp2t is a frequency-modulated US signal identical to Chirp2r, which is digitally synthesized by the FPGA of NODE2. The duration as well as the operating frequency bands of Chirp2r and Chirp2t are equal and reside in the frequency band of PZT2. The only difference is that Chirp2r starts at the very moment that the ranging is initiated, while the response (Chirp2t) arriving from NODE2 is delayed by the round trip time of US. The delay $\tau_{d}$ in Fig. $1 \mathrm{~b}$ and Fig. 2 corresponds to the round trip time of flight between the nodes and equals to $\frac{2 R}{c_{U S}}$, where $R$ is the distance between the nodes and $c_{U S}$ is the speed of US in the medium. NODE1 records the signal obtained after mixing Chirp2r and Chirp2t in MAINRX to its memory. As shown in Fig. 2, the spectrum of this signal will show a frequency peak, $f_{\text {beat }}$, corresponding to the time of flight $\tau_{d}$ of US traveling back and forth between NODE1 and NODE2. An important highlight of the proposed method is that the initial frequency, $f_{1}$, the stop frequency, $f_{2}$, as well as the duration, $T_{\text {sweep }}$, of the identical Chirps are known apriori and digitally synthesized in the nodes to fit inside the bandwidth of PZT2. As a result, the relative distance between NODE1 and NODE2 can be calculated with the knowledge of BW, $T_{\text {sweep }}$, and $c_{U S}$, as shown in Fig. 2. Since the proposed measurement method is only controlled by the initiator node, a global synchronization between the nodes is not needed. Clearly, the clocks needed for digital synthesis in the initiator and the wake-up nodes should be sufficiently accurate during the time $T_{\text {sweep }}$. However, this requirement is easy to achieve considering the rather short duration of $T_{\text {sweep }}$ (which is typically in the order of milliseconds) and a typical short-term accuracy of real time clocks (RTCs) [20], which is better than $100 \mathrm{ppm}$. A $100 \mathrm{ppm}$ variation in the RTCs used in the nodes would translate an error in $T_{\text {sweep }}$ that is lower than $0.01 \%$, which will thus not affect in an appreciable way the resolution of the ranging system. Thanks to the different frequency bands exploited for the wake-up 


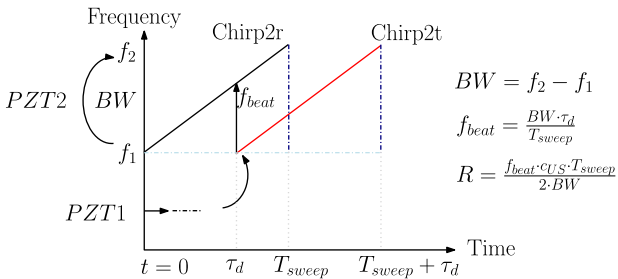

Fig. 2. Illustration of the proposed distance $(\mathrm{R})$ measurement method in a frequency-time plot.

signal $W U R X_{\text {code }}$ sent by the initiator and the FMCW US signal sent back by the nodes that wake-up, the initiator node can distinguish echoes coming from passive reflectors from frequency-modulated response signals sent back by the nodes. This happens even if the wake-up nodes are approximately at a fixed distance from the initiator, and thus if no Doppler shift occurs. One should notice, indeed, that in the applications envisaged, the swarm of nodes is moving with the flow of the fluid, therefore the relative distances between the nodes are expected to change slowly in time.

Furthermore, in case multiple sensor nodes that are at different distances from NODE1 wake-up, their response will correspond to different beat frequencies that are separated in the frequency domain, and can be distinguished during post-processing [21]. Finally, an identification (ID) payload of the woke-up node can be embedded in the response Chirp2t, for instance by amplitude modulation [22] or phase modulation [23]. This allows distinguishing between the first signal received by a node that wakes up and possible delayed version of the signal coming from the same node due to multipath propagation [21].

\section{B. Transducer Characterization}

Most of the applications considered in this paper use a fluidic medium, where the US propagates with much lower attenuation $(<100 \times$ in water [24]) compared to air. However, due to the difficulty to measure at large distances in fluids, and thus to simplify the characterizations while keeping the maximum distance between the sensor nodes at a manageable level, the experimental setup of the proposed system has been built in air to demonstrate the proof-of-concept principle. Piezoelectric transducers (PZTs) have been used because of their availability in the market. PZTs typically have a nominal operating frequency spanning from a few tens of $\mathrm{kHz}$ to several hundreds of $\mathrm{kHz}$ with a relatively low bandwidth [25], [26]. Although Capacitive Micromachined Ultrasound Transducers (CMUTs) have been used in literature [27] to achieve a larger bandwidth compared to PZTs, the high-voltage DC bias requirement of CMUTs [28] is an important drawback in battery-operated applications. Accordingly, PZT1 and PZT2 are chosen as commercially available piezoelectric transducers with a nominal resonance frequency of $40 \mathrm{kHz}$ and $100 \mathrm{kHz}$, and an input capacitance of $2 \mathrm{nF}$ and $1 \mathrm{nF}$, respectively [29]. Fig. 3a shows an impedance measurement of PZT1, where its resonance frequency and the impedance at resonance are measured as $39 \mathrm{kHz}$ and $400 \Omega$, respectively. Fig. $3 \mathrm{~b}$ shows the normalized received power for a link consisting of two identical PZT2 transducers, where the
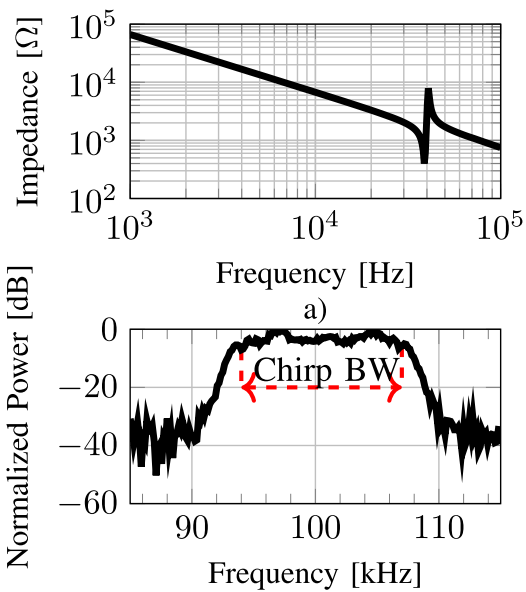

b)

Fig. 3. (a) Measured impedance plot of PZT1. The series resonance is at $39 \mathrm{kHz}$. (b) A link characterization from PZT2 to PZT2, where a bandwidth of $13 \mathrm{kHz}$ between $94 \mathrm{kHz}$ and $107 \mathrm{kHz}$ is found suitable for the Chirp signals.

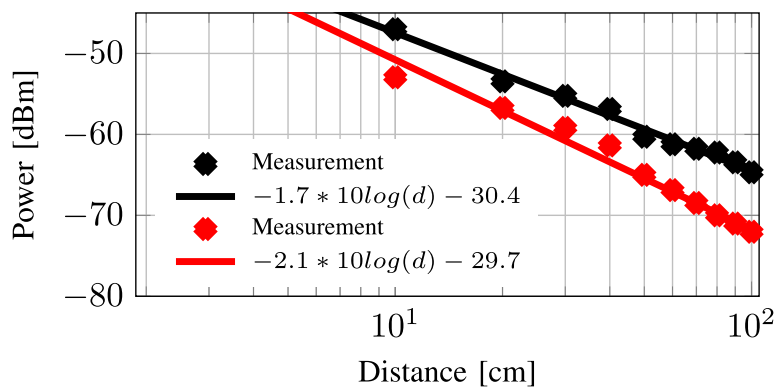

Fig. 4. Received power characterization in air for PZT2 (black, top) and PZT1 (red, bottom)

frequency band between $94 \mathrm{kHz}$ to $107 \mathrm{kHz}$ has been chosen for the Chirp signals. PZT2 is used in the FMCW ranging link, as a larger bandwidth in the Chirp signals is desired to improve the theoretical range resolution [21]. The resonance frequency of PZT1 is used as a carrier frequency in the wake-up link to broadcast the $W U R X_{\text {code }}$.

\section{Link Budget Analysis and System Specifications}

Power-loss in the propagation of US is investigated by building a test setup in air at room temperature, where PZT1 and PZT2 are driven with a $0.6 \mathrm{~V}_{\mathrm{pp}}$ burst signal at $39 \mathrm{kHz}$ and $100 \mathrm{kHz}$, respectively, and the power received by an identical transducer is recorded up to $1 \mathrm{~m}$ distance. Fig. 4 shows this characterization, where the received power is modeled with the equation: $P_{r e c}=-k \cdot 10 \log (d)+P_{0}$. Here, $d$ is the distance in $\mathrm{cm}, k$ is the path loss coefficient, and $P_{0}$ is the initial power loss. The outcome of this characterization is reported in Fig. 4, where the received powers of PZT1 and PZT2 at $1 \mathrm{~m}$ distance are measured as $-72 \mathrm{dBm}$ and $-65 \mathrm{dBm}$, respectively. Although PZT1 has a lower resonance frequency, it is less directive compared to PZT2, which leads to a larger signal attenuation with distance. While it is possible to balance the received power of the links, for instance by reducing the driving voltage of PZT2, we have chosen to use a single $0.6 \mathrm{~V}$ driving supply to avoid using an extra voltage regulator. At the input of the receivers $\approx 12 \mathrm{~dB}$ SNR is sufficient to have a $90 \%$ detection probability with a $<10^{-3}$ false alarm ratio [30]. 
TABLE I

SYSTEM SPECS

\begin{tabular}{ll}
\hline Parameter & Value \\
\hline$V_{D D}$ & $0.6 \mathrm{~V}$ \\
Max. distance in air & $\approx 1 \mathrm{~m}$ \\
$S N R_{\text {in }}$ & $>12 \mathrm{~dB}$ \\
$I R N_{W U R X}$ & $<14 \mu \mathrm{V}_{\text {rms }}$ \\
$B W_{W U R X}$ & $\approx 1 \mathrm{kHz}$ \\
$I R N_{M A I N R X}$ & $<32 \mu \mathrm{V}_{\text {rms }}$ \\
$T_{\text {sweep }}$ & $10 \mathrm{~ms}$ \\
$f_{\text {beat }}$ & $<7.6 \mathrm{kHz}$ \\
BW $W_{\text {MAINRX }}$ & $>10 \mathrm{kHz}$ \\
\hline
\end{tabular}

As summarized in Table I, this can be achieved when the integrated Input-Referred Noise (IRN) of the WURX and MAINRX is lower than $14 \mu \mathrm{V}_{\text {rms }}$ and $32 \mu \mathrm{V}_{\text {rms }}$, respectively. To reduce the always-on power consumption of the nodes, a low-complexity communication scheme (e.g. On Off Keying) with a limited data-rate (e.g $\leq 0.5 \mathrm{kbps}$ ) can be exploited in the wake-up link [31]. This allows allocating a $\approx 1 \mathrm{kHz}$ baseband bandwidth $\left(B W_{W U R X}\right)$, thus an Input-Referred Noise Density (IRND) of $443 \frac{n V}{\sqrt{H z}}$ for the WURX is estimated. The duration of the Chirp signals, $T_{\text {sweep }}$, has to be larger than the round trip time $(\approx 6 \mathrm{~ms})$ among the sensors at the maximum specified measurement distance of $1 \mathrm{~m}$ in air, therefore, $T_{\text {sweep }}$ is chosen to be $10 \mathrm{~ms}$. The frequency band between $94 \mathrm{kHz}$ and $107 \mathrm{kHz}$ was chosen for the FMCW link, thus a $13 \mathrm{kHz}$ Chirp $\mathrm{BW}$ is used in the ranging. Accordingly, the smallest distance step that can be measured $\left(R_{\min }=\frac{c_{U S}}{2 B W}\right)$ in air is calculated as $13 \mathrm{~mm}$ [21]. At $1 \mathrm{~m}$ distance, an $f_{\text {beat }}$ of $\approx 7.6 \mathrm{kHz}$ is estimated, which has to reside inside the $\mathrm{BW}$ of the MAINRX $\left(B W_{M A I N R X}\right)$. Therefore, by specifying at least $10 \mathrm{kHz} \mathrm{BW}$, an IRND of $320 \frac{n V}{\sqrt{H z}}$ for the MAINRX is calculated.

\section{High LeVEl Description of HaRdware Blocks}

US transducers can typically be modeled [32] by a series resonator $\left(R_{m}, L_{m}, C_{m}\right)$ in parallel with a parasitic capacitor, $C_{p}$, as shown in Fig. 5a. When driving piezoelectric transducers, a large amount of energy is dissipated to charge and discharge the large parasitic capacitance $C_{p}$. Using an inductance (mH range) to cancel out $C_{p}$ requires large volumes and is thus impractical. To reduce capacitive losses, a well-known step-wise charging method [26], [33], [34] has been used in literature. In this work, a similar method is chosen to drive the transducers PZT1 and PZT2. For each transducer, a stepwise charging/discharging using a multi-level driving signal is more energy-efficient than the conventional two-level driving scheme. On one hand, multi-level driving reduces the losses associated to charging and discharging the parasitic transducer capacitance. On the other hand, multi-level periodic signals have a higher ratio between the energy at the fundamental frequency and the energy in the harmonics. For this reason, multi-level signals can excite more efficiently the resonator branch of the transducer and transform electric energy into acoustic one. To realize an efficient transmitter, thus, a multilevel TX is used in this work, as shown in Fig. 5b. A 4-level driving signal has been chosen, which is generated connecting the transducer periodically to $V D D_{T X}$, the maximum voltage level applied to the load, two intermediate voltage levels $(2 / 3$. $V D D_{T X}$ and $1 / 3 \cdot V D D_{T X}$ ), and ground. The intermediate

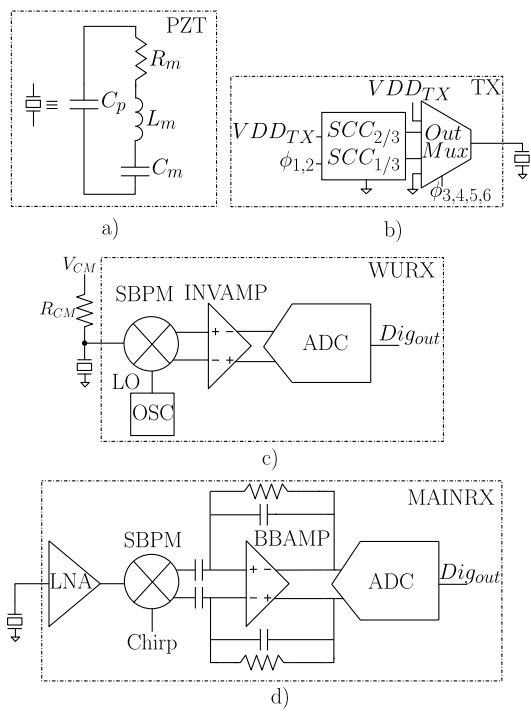

Fig. 5. a) Equivalent circuit model of a PZT transducer. b) Top-level circuit implementation of TX, c) WURX, d) MAINRX.

DC voltage levels are obtained from $V D D_{T X}$ by using two Switched-Capacitor Converters $\left(S C C_{1 / 3,2 / 3}\right)$. These converters use two $50 \%$ duty-cycled non-overlapping clocks, $\phi_{1,2}$. Each DC level is connected to the transducer for a time span and in an order that are controlled via the output multiplexer by the clocks $\phi_{3, . .6}$.

As shown in Fig. 5c, a mixer-first topology with a single-balanced passive mixer (SBPM) is used in the WURX to minimize the always-on power consumption of the nodes. Although the input-referred noise density of the front-end increases without an LNA preceding the mixer, the target integrated noise level is achievable with this topology by keeping a limited BW of $1 \mathrm{kHz}$ in the baseband amplifier. This is possible, as the transducer PZT1 is used at its resonance frequency, and only the fundamental component is needed to transmit the wake-up message. The SBPM does not consume DC power, but enables single-ended to differential conversion, thus providing a theoretical gain of $1.48 \mathrm{~dB}$ [35]. After the SBPM, the WURX comprises an open-loop inverter-based baseband amplifier, INVAMP, and a 10-bit SAR ADC, which uses a similar design as reported in [36]. The down-conversion signal of the SBPM, $L O$, as well as the ADC clock, are provided by an on-chip oscillator, $O S C$. A single supply, $V_{D D}$, has been used in the WURX. The transducer is DC coupled to the front-end, and the DC common-mode bias of the front-end, $V_{C M}=V_{D D} / 2$, is provided through an off-chip resistance, $R_{C M}$, of $5 \mathrm{M} \Omega$.

The MAINRX exploits a basic heterodyne architecture as shown in Fig. 5d, which comprises a single-ended inverterbased low-noise amplifier (LNA), followed by an SBPM, a closed-loop baseband amplifier (BBAMP), and a 10-bit SAR $\mathrm{ADC}$, all sharing the same supply, $V_{D D}$. The Chirp needed for mixing is provided by the digital backend as a two-level square wave. The high-frequency harmonics of the SBPM are filtered out by the BBAMP, and the baseband signal is converted to the digital domain by the 10-bit SAR ADC [36]. A detailed report on the MAINRX can be found in [7], while some parts of this circuit are briefly discussed here for better readability. A Xilinx 

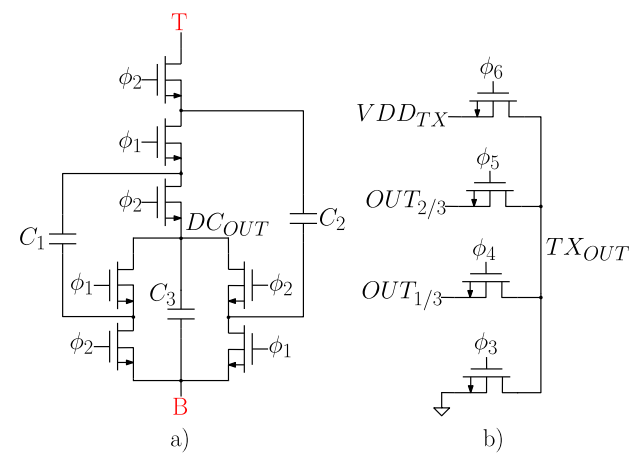

Fig. 6. Schematic of the a) Dickson converter, b) Output multiplexer.

Spartan-3 FPGA Board has been used as digital backend. The Chirp needed in the MAINRX and the output multiplexer control clocks of the TX, $\phi_{3, . .6}$, are synthesized by reading at a constant rate $(1 \mathrm{MHz})$ suitable bit streams preloaded to a memory in the digital backend. The time reference is provided by an external $50 \mathrm{MHz}$ crystal oscillator available in the FPGA board, which in wake-up mode can be duty cycled using the wake up signal. All other timing signals needed in the nodes are derived from the same reference, except for the always active clocks in wake-up mode, which are directly derived from the on-chip oscillator OSC. Most of the digital signal processing, such as the FFT needed to determine the beat frequency in the FMCW ranging, are performed outside of the nodes. The output data generated from the MAINRX by mixing the Chirp signals during the ranging are thus saved to a memory in the digital backend.

\section{iV. Circuit Design}

\section{A. TX Circuit Design}

An SCC can be modeled [37] as an ideal transformer (whose voltage ratio is defined by the SCC topology) in series with an output resistor, $R_{\text {out }}$. The DC level of the output of the TX is determined by the voltage division between $R_{\text {out }}$ and the load impedance. In our implementation, $R_{\text {out }}$ represents the output resistance of the corresponding SCCs with an additional series resistance due to the output multiplexer (Fig. 5b). To reduce the power consumption of the TX, $0.6 \mathrm{~V}$ is chosen as $V D D_{T X}$. Each clock phase $\phi$ is buffered to the switching transistors in the SSCs and output multiplexer by on-chip drivers, which use a $V D D_{\text {driver }}=1.2 \mathrm{~V}$ supply. The transistor-level implementation of the TX is shown in Fig. 6. All switches are implemented using insulated well nMOS transistors. A Dickson topology is chosen to implement the SCCs, since the same topology can be reused to obtain the intermediate voltage levels with a minor change in top-level connection. As shown in Fig. 6a, a voltage conversion ratio of 3:1 is obtained when $V D D_{T X}$ and ground are connected to the top node, T, and the bottom node, B, respectively. The division ratio of 3:2 is obtained by interchanging the top node and the bottom node. The SCC output node is marked $D C_{\text {out }}$ in Fig. 6a. $O U T_{1 / 3}$ and $O U T_{2 / 3}$ correspond to the output of $S C C_{1 / 3}$ and $S C C_{2 / 3}$, respectively. The capacitors $C_{1,2,3}$ are off-chip flying capacitors. The output multiplexer comprises 4 NMOS transistors that connect the desired DC voltage to the transducer load, as shown in Fig. 6b. An NMOS

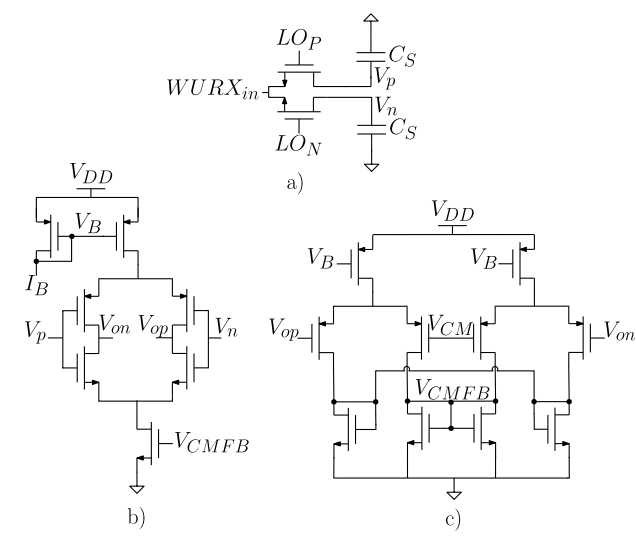

Fig. 7. Building blocks of the WuRX; a) Single-Balanced Passive Mixer, b) Inverter based baseband amplifier, c) Its CMFB.

transistor is chosen to build the switches due to its lower combined conduction and switching losses compared to a PMOS transistor with a similar overdrive voltage. A deepNWELL transistor with a size of $10 \mu \mathrm{m} / 0.3 \mu \mathrm{m}$ and 32 fingers is used as a unit switch in $S C C_{1 / 3}$. In $S C C_{2 / 3}$ and the output multiplexer, a parallel connection of four of these unit switches is used. This improves the driving efficiency of the TX as a lower SCC output resistance is assigned to a higher DC voltage level. The value of the flying capacitors is chosen as $1 \mu \mathrm{F}$, which leads to a simulated corner frequency between Fast and Slow Switching limits [37] of $10 \mathrm{kHz}$. The simulated total output resistance is $20 \Omega$ and $10 \Omega$ for $S C C_{1 / 3}$ and $S C C_{2 / 3}$ in the Fast Switching Limit, respectively.

\section{B. WuRX Circuit Design}

Fig. 7 shows the transistor-level implementation of the WURX. The SBPM (Fig. 7a) comprises two NMOS transistors and sampling capacitors, $C_{S}$. The size of the transistors $(400 \mathrm{~nm} / 60 \mathrm{~nm})$ is chosen to reduce the dynamic power. A $C_{S}$ of $8.5 \mathrm{pF}$ is used to lower the noise contribution of the mixer. Considering the large capacitance of the PZT transducers, the loading introduced by these capacitors is negligible. Fig. 7b and Fig. 7c show the implementation of the inverter-based baseband amplifier and its common-mode feedback (CMFB) [38], respectively. An inverter-based topology is chosen to increase the power efficiency while all transistors are biased in the weak-inversion region with a $g_{m} / I_{D}$ ratio of $31 \mathrm{~V}^{-1}$. With a supply voltage, $V_{D D}$, of $0.6 \mathrm{~V}$, a bias current, $I_{B}$, of $0.35 \mathrm{nA}$, and an $\mathrm{LO}$ of $38 \mathrm{kHz}$, the post-layout simulations of the front-end predict a total conversion gain of $34 \mathrm{~dB}$, a $1.2 \mathrm{kHz} 3-\mathrm{dB}$ BW and an IRND of $365 \mathrm{nV} / \sqrt{\mathrm{Hz}}$. Thus, the IRN is calculated as $13 \mu \mathrm{V}_{\mathrm{rms}}$, which satisfies the specs in Table I. The digital output of the WURX will be followed by a digital thresholding, to decide whether or not to wake-up the node. Although the sensitivity of the front-end can be degraded due to the variations of the LO over PVT, the digital thresholding will not be influenced, and the nodes can successfully wake-up, as long as the SNR at the input of the front-end is sufficient. Thereby, the accuracy requirement of the LO frequency can be relaxed to save power.

This allows using a simple on-chip clock generator, as shown Fig. 8. Here, an external reference current, $I B_{C L K}$, is integrated on the capacitor, $C_{R}$, to generate a ramp voltage. 


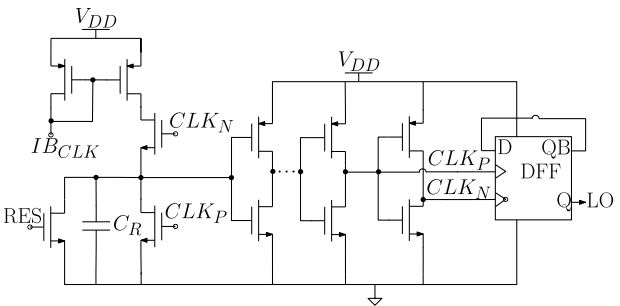

Fig. 8. Schematic of the on-chip clock generator in WURX (OSC).

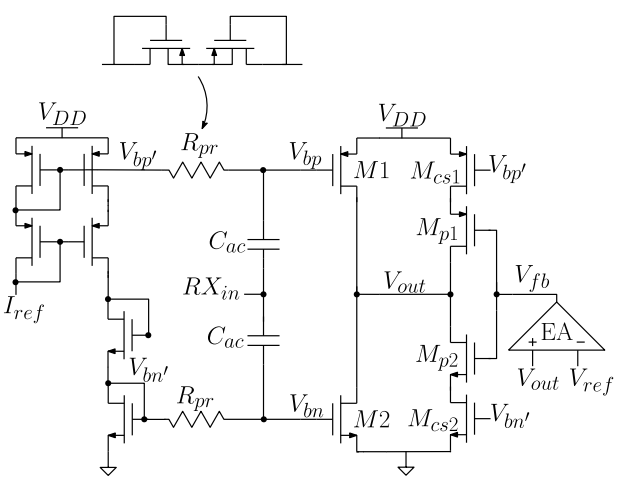

Fig. 9. Schematic of the LNA in MAINRX [7].

Then, a feedback loop comprising 6 stages of inverters resets the voltage on $C_{R}$ when the threshold of the first inverter is reached. An external reset signal, $R E S$, is used only at startup. High-threshold transistors are used in the inverter chain to limit the power consumption, while 6 stages of inverters are used to provide enough on-time for the generated pulse signal. To obtain a $50 \%$ duty-cyle LO, the inverter chain is followed by a Master/Slave D-type flip-flop, which is implemented with two pass gate latches. The Q output of the DFF is used as an input to the non-overlapping clock generator circuit [39], which provides the signals, $L O_{P}$ and $L O_{N}$, used by the SBPM for down-conversion. The ADC clock is generated dividing by two the LO.

\section{MAINRX Circuit Design}

The MAINRX uses a single-ended inverter-based LNA to save power. A DC feedback is needed to stabilize its output DC voltage. As shown in Fig. 9, a current starved auxiliary inverter, formed by $M_{p 1}$ and $M_{p 2}$, is used in parallel to the main inverter $\left(M_{1}\right.$ and $\left.M_{2}\right)$ to stabilize the DC level of the output node, $V_{\text {out }}$. The gate of the auxiliary inverter is controlled via feedback by comparing $V_{\text {out }}$ to a reference voltage, $V_{r e f}$, and feeding back the error signal, $V_{f b}$, amplified by the error amplifier, EA [7]. The auxiliary inverter can either source or sink a suitable current to the output node, thus keeping the output DC voltage at the desired value. The proposed DC feedback can compensate the errors due to leakage of pseudo-resistors, $R_{p r}$, and mismatch between the biasing network and the LNA. The transconductance of the auxiliary inverter is much lower than the main inverter. This provides a small DC current, low power, and high output resistance, minimizing the impact of the auxiliary inverter on the LNA gain. Compared to previous works [32], [40], [41], the proposed DC feedback allows the single-ended inverter

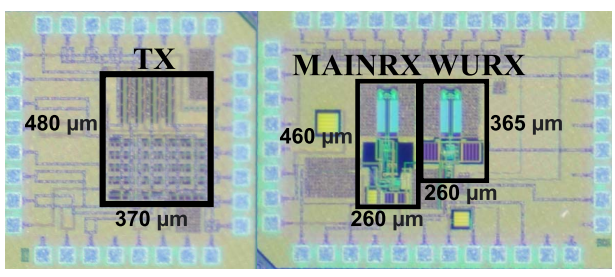

Fig. 10. Die photo of the transmitter TX and receivers: MAINRX and WURX.

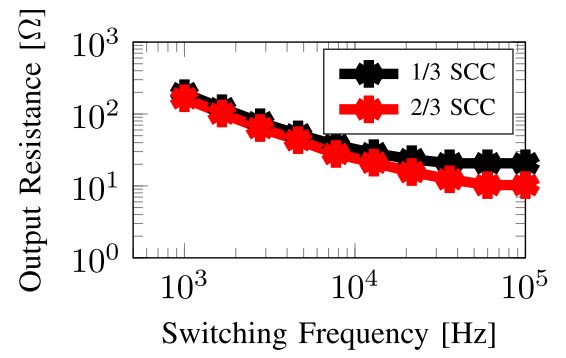

Fig. 11. Measured output resistance of the switched capacitor converters (SCC) with respect to the switching frequency.

to work in continuous-time. Besides, it applies the feedback to the output node instead of the LNA input, minimizing degradation of the SNR. The SBPM uses similar size transistors as in the WURX, however, the sampling capacitors are not used to avoid loading the LNA. Due to the presence of the LNA in the MAINRX, the resulting $\mathrm{kT} / \mathrm{C}$ noise of the mixer is negligible when referred to the input. The BBAMP uses a two-stage architecture with Miller compensation. The feedback capacitors have a ratio of $(134 \mathrm{fF} / 35 \mathrm{fF})$ to obtain a gain of about $12 \mathrm{~dB}$. The complete front-end is simulated to have a conversion gain of $43 \mathrm{~dB}$, a $3-\mathrm{dB} \mathrm{BW}$ of $20 \mathrm{kHz}$, and an IRN of $7.8 \mu \mathrm{V}_{\text {rms }}$ while using a single $0.6 \mathrm{~V}$ supply. The MAINRX can be overdesigned in terms of IRN and 3-dB BW compared to the specs in Table I, as the MAINRX is normally off, and a larger bandwidth can be useful to improve ranging resolution in means characterized by higher speed of sound.

\section{Measurement Results}

A $65 \mathrm{~nm}$ CMOS process is used for fabrication and the die photos of the circuits are shown in Fig. 10. A detailed measurement of the individual circuits and the proposed ranging system will be presented in the following sections.

\section{A. TX Characterization}

To characterize the output resistance of the TX, the output voltage of each SCC is measured with an external $400 \Omega$ resistor load (equal to the impedance of PZT1 at resonance) while varying the switching frequency. The outcome is depicted in Fig. 11, where the two asymptotes of Slow Switching Limit (SSL) and Fast Switching Limit (FSL) [37] can be appreciated. The transient output voltage of the TX is shown in Fig. 12, where a 4-level output driving signal with a frequency of $40 \mathrm{kHz}$ is shown while switching the SCCs at $10 \mathrm{kHz}$. The performance summary of the TX when PZT1, PZT2, and a $10 \mathrm{nF}$ capacitor are used as a load is reported in Table II. In these measurements, $V D D_{T X}=0.6 \mathrm{~V}$, while 


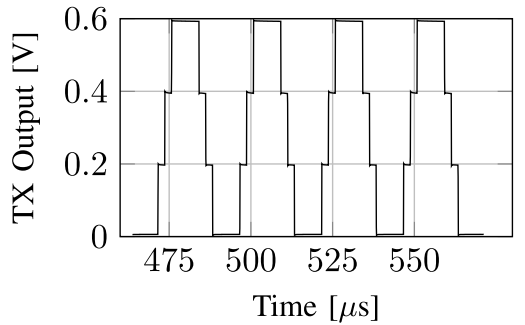

Fig. 12. Measured four-level output voltage of the TX with a $400 \Omega$ load.

TABLE II

TX CHARACTERIZATION SUMMARY

\begin{tabular}{cccc}
\hline & PZT1 & PZT2 & $10 \mathrm{nF}$ \\
\hline Driving Frequency [kHz] & 39 & $94-107$ & 39 \\
SCC Switching Frequency [kHz] & 10 & 10 & 3 \\
$V D D_{\text {driver }[\mathrm{V}]}$ & 1.2 & 1.2 & 1.2 \\
$I D C_{d r i v e r}[\mu A]$ & 3.2 & 3.2 & 1.07 \\
$V D D_{T X}[\mathrm{~V}]$ & 0.6 & 0.6 & 0.6 \\
$I D C_{4-\text { level }}[\mu A]$ & 231.7 & 131.1 & 79.8 \\
$I D C_{2-l e v e l}[\mu A]$ & 311.1 & 210.4 & 230.1 \\
Improvement: $\frac{I D C_{2}-I D C_{4}}{I D C_{2}}$ & $25.5 \%$ & $37.7 \%$ & $65.3 \%$ \\
\hline
\end{tabular}

the gate drivers are supplied by $V D D_{\text {driver }}=1.2 \mathrm{~V}$, PZT1 is driven at its resonance frequency of $39 \mathrm{kHz}$, the capacitor is driven at the same frequency, while PZT2 is driven with Chirp2t. The average currents, $I D C$ and $I D C_{\text {driver }}$, drawn from the supply voltages, $V D D_{T X}$ and $V D D_{\text {driver }}$, respectively, are measured. A load of $10 \mathrm{nF}$ is used as a reference to validate the energy-saving in the case that the load is purely capacitive. As summarized in Table II, a reduction of $65.3 \%$ in $I D C$ is measured when a 4-level driving signal is applied to the $10 \mathrm{nF}$ load compared to the two-level case. This measurement is in good agreement with the fact that a step-wise charging of a capacitor in three equal steps reduces the energy spent to charge the capacitor to one third of the one needed when charging the capacitor directly to the final voltage level [33]. Concerning the experiments with PZT1, this transducer is driven at its resonance and an identical PZT1 is placed at $5 \mathrm{~cm}$ apart to monitor the received signal. In this experiment, a reduction of $25.5 \%$ in $I D C$ when using four levels instead of two is observed, while there is only a $1.4 \%$ loss in the received signal power, compared to the two-level case. This is due to the fact that four-level driving at the same driving frequency as the two-level one contains less energy in the harmonics, while having a similar energy at the fundamental tone. Moreover, a relatively larger reduction of $37.7 \%$ in IDC is measured when driving PZT2 with Chirp2t and using four levels instead of two. As the driving frequency is swept in a BW of $13 \mathrm{kHz}$, the impedance of PZT2 is in average more capacitive compared to that experienced in the measurement of PZT1, and thus a larger energy-saving is measured than the one experienced in the measurement of PZT1 at resonance. The electrical efficiency of the TX is defined as the ratio between the electric power delivered to the transducer and the total power consumed by the TX. The measured TX efficiency when driving PZT1 with a four-level driving signal at its resonance frequency of $39 \mathrm{kHz}$ is $88 \%$. In resonance, part of the power losses come from the resistance of the switches

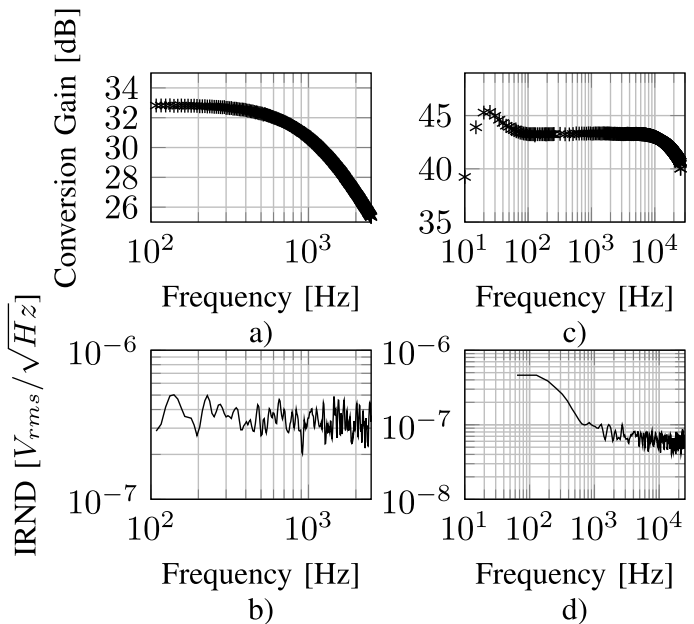

Fig. 13. (a) Measured conversion gain of the WURX for an $\mathrm{LO}=39 \mathrm{kHz}$. The maximum gain is $32.8 \mathrm{~dB} @ 200 \mathrm{~Hz}$ and the $3-\mathrm{dB}$ BW is $1.2 \mathrm{kHz}$. (b) Corresponding IRND of the WURX. IRND = $400 n V_{r m s} / \sqrt{H z} @ 1.2 \mathrm{kHz}$ is measured. (c) Measured conversion gain of the MAINRX for an $\mathrm{LO}=100 \mathrm{kHz}$. The gain is $43.3 \mathrm{~dB} @ 5 \mathrm{kHz}$ and the $3-\mathrm{dB} \mathrm{BW}$ is from $10 \mathrm{~Hz}$ to $25 \mathrm{kHz}$. (d) Corresponding IRND of the MAINRX. IRND =60nV $V_{r m s} / \sqrt{H z} @ 5 \mathrm{kHz}$ is measured.

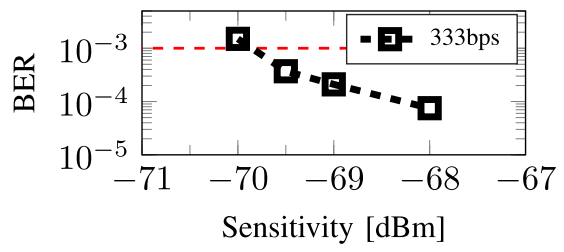

Fig. 14. Measured BER of the WURX.

and part of the losses come from switching on and off the switches. Out of resonance, the efficiency will decrease, as the load becomes capacitive. Indeed the measured transmitter efficiency when driving PZT2 with a two-level Chirp signal $(94 \mathrm{kHz}-107 \mathrm{kHz})$ is $47 \%$, and the efficiency increases to $62 \%$, when a four-level Chirp signal is used.

\section{B. WURX and Wake-Up Link Characterization}

1) Electrical Measurements: The measured conversion gain and IRND of the WURX for an LO $=39 \mathrm{kHz}$ are shown in Fig. 13a and Fig. 13b, respectively. The total integrated noise in the $3-\mathrm{dB} \mathrm{BW}$ of $1.2 \mathrm{kHz}$ is found to be $14 \mu \mathrm{V}_{\mathrm{rms}}$. For these measurements, external reference currents, $I_{B}$ of $0.35 \mathrm{nA}$ and $I B_{C L K}$ of $0.45 \mathrm{nA}$, are used. The sensitivity level of the WURX is estimated first with electrical BER measurements, where an OOK modulated signal with different attenuated amplitudes is provided by an arbitrary signal generator to the WURX, and the ADC codes at the output are read-out and transferred to MATLAB for demodulation. Fig. 14 shows BER plots for this setup, where a $-69.5 \mathrm{dBm}\left(74.9 \mu \mathrm{V}_{\mathrm{rms}}\right)$ input signal at $333 \mathrm{bps}$ data-rate is demodulated with a $10^{-3}$ BER. This sensitivity level is validated with an experimental setup built with two PZT1 transducers, a TX, and a WURX to transmit OOK signals using a $39 \mathrm{kHz}$ carrier. An input signal of $-69.5 \mathrm{dBm}$ is received when the transducers are $75 \mathrm{~cm}$ apart and a successful communication link with a $10^{-3}$ BER is established in air. In this measurement, the on-chip oscillator is used to provide a $38 \mathrm{kHz} \mathrm{LO}$. The resulting $1 \mathrm{kHz}$ baseband signal is sampled with a $19 \mathrm{kHz}(\mathrm{LO} / 2)$ ADC sampling clock. 


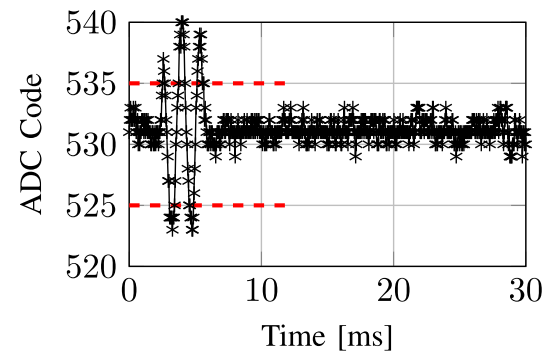

Fig. 15. A Measurement of WURX ADC code when TX transmits a single one (encoded as 2.5 periods of sinusoid) as $W U R X_{C O D E}$.

Although lowering the sampling frequency will reduce the ADC power consumption, a $19 \mathrm{kHz}$ sampling clock is used to have sufficient margin to sample the baseband signal considering the temperature sensitivity of the LO, as will be discussed in the following section.

2) Missed Detection Rate (MDR) Measurements: In order to reduce the demodulation complexity in the digital domain and to save power, the WURX digital output is used in a digital threshold detector, where a threshold around the DC output code of the ADC is applied to wake-up the node. The measurement shown in Fig. 15 is performed when NODE1 and NODE2 are $75 \mathrm{~cm}$ apart, and NODE2 activates its TX to broadcast the $W U R X_{\text {code }}$ of a symbol one, ' 1 '. Since the initial phase of two separate nodes is unknown, two different thresholds have been chosen; an upper one (535) and a lower one (525) to detect the level of the incoming signal. The ratio between the threshold levels and the rms of the noise voltage at the output of the WURX determines the probability of a false alarm. To have a false alarm rate of one per 15min (the average time interval between the crossings of the threshold due to noise), the threshold levels are chosen as $\approx 4$ times the rms value of the measured noise voltage $(\sim 0.8 L S B)$ at the output of the WURX [30]. To characterize the performance of the WURX link, missed-detection rate (MDR) measurements have been performed, too. In these measurements, the data rate is set to $100 \mathrm{bps}$, where the symbol '1', as shown in Fig. 15, is transmitted in a $10 \mathrm{~ms}\left(=T_{\text {sweep }}\right)$ period, according to the proposed system. Lowering the data rate compared to our measurements in Fig. 14 relaxes the SNR needed to correctly receive a ' 1 ', and enables increasing the achievable distance for $10^{-3}$ MDR between the nodes to $1 \mathrm{~m}$ in air, satisfying the specs in Table I. To further investigate the WURX link performance under temperature variation, the WURX is placed in a temperature chamber and the operating temperature of the WURX is swept from $0{ }^{\circ} \mathrm{C}$ to $80^{\circ} \mathrm{C}$. During these characterizations, the external bias currents $\left(I B_{C L K}\right.$ and $\left.I_{B}\right)$ provided to the WURX are also varied with the temperature according to the data reported for the reference current generator in [42]. The results of these experiments are summarized in Fig. 16, where at room temperature of $27^{\circ} \mathrm{C}$, the wake-up link has been established until $1 \mathrm{~m}$ in air with an MDR lower than $10^{-3}$. When the operating temperature increases, the clock frequency of the on-chip oscillator increases as well. This results in that the baseband signal after down-conversion falls outside the 3-dB bandwidth of the WURX, worsening the sensitivity of the front-end. When the temperature is reduced to $0^{\circ} \mathrm{C}$,

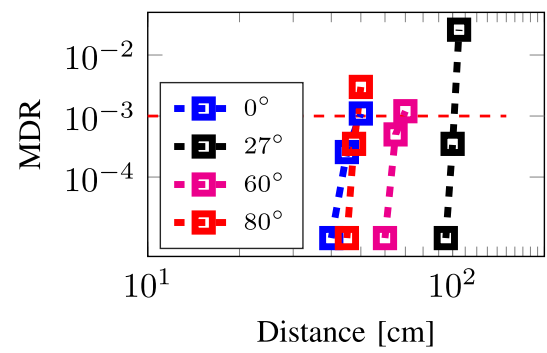

Fig. 16. MDR characterization of WURX.

the LO frequency and the bandwidth of the amplifier decrease together, thus the baseband signal is attenuated even more and further degrades the sensitivity. As shown in Fig. 16, at the limit temperatures of $0^{\circ} \mathrm{C}$ and $80^{\circ} \mathrm{C}$, the distance that the WURX link can achieve with an MDR better than $10^{-3}$ is reduced to $45 \mathrm{~cm}$. For these temperatures, the power supply can be increased by $10 \%$ while keeping an MDR better than $10^{-3}$. It is important to mention that in [42] about $+-30 \%$ change of the absolute value of the reference current due to process variations is reported. In the WURX link, it is desired to keep the baseband signal, obtained after down converting the carrier $(39 \mathrm{kHz})$ of the $W U R X_{\text {code }}$ with the LO, inside the $3-\mathrm{dB} \mathrm{BW}$ of the amplifier $(1.2 \mathrm{kHz})$, to maintain the sensitivity of the front-end. This means that a calibration of the $\mathrm{LO}$ with at least 5-bit resolution is needed to keep the LO error e.g. within $2 \%$ from its $39 \mathrm{kHz}$ target. Such calibration, which can be implemented with a simple current DAC fed by the current reference, is not yet implemented in the present chip.

At room temperature, a total power consumption of $23.1 \mathrm{nW}$ for the WURX under a single supply of $0.6 \mathrm{~V}$ is measured. This figure includes the power of on-chip buffers used to distribute the $\mathrm{LO}(38 \mathrm{kHz})$ and the ADC sampling clock (LO/2). The comparison of the ADC code with the thresholds is done in the FPGA by using simple digital comparators, where the output of the on-chip clock generator is used as a clock. The power overhead of this comparison is simulated at a transistor level as $0.5 \mathrm{nW}$, and added to the power consumption of the WURX. As a result, a $23.6 \mathrm{nW}$ power consumption is obtained. Although the power required to generate the reference currents is excluded from this figure, this overhead is estimated to be much lower $(120 \mathrm{pW})$ than the reported WURX power, when using relevant prior-art implementations in similar technology and supply voltage [42]. A distance of $45 \mathrm{~cm}$ in air at the worst-case operating conditions is sufficient in the foreseen applications, indeed, considering that the attenuation of US in water is more than 100 times lower than that of in air [24], a much larger operating distance can be obtained if the nodes are operated in a fluidic environment. In water, the limiting factor for the distance measurement will be the sweep time of the Chirps, which must be longer than the round-trip time of flight between nodes. Considering that the speed of US in water is around $1500 \mathrm{~m} / \mathrm{s}$, the max. achievable distance in fluids with a $T_{\text {sweep }}$ of $10 \mathrm{~ms}$ is calculated to be $7.5 \mathrm{~m}$. If a larger distance in fluids is needed, a longer sweep time can be used at the cost of an increased energy consumption during the ranging. 


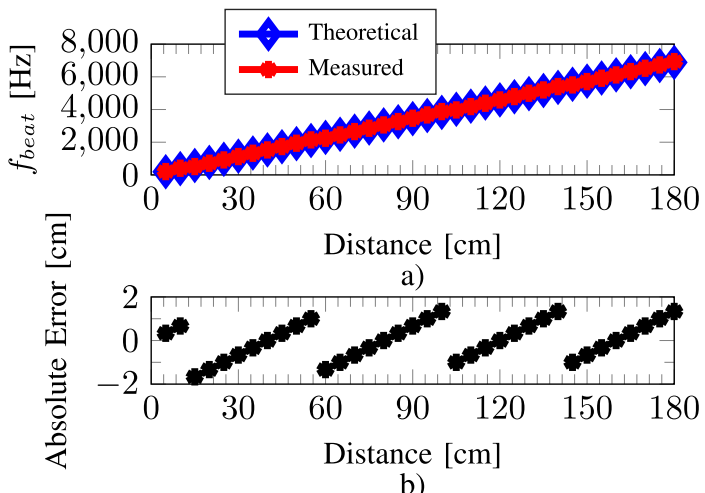

b)

Fig. 17. a) FMCW ranging link characterization. b) Absolute error in the distance calculation.

\section{MAINRX and Ranging Link Characterization}

1) Electrical Measurements: The measured conversion gain and IRND of the MAINRX for an LO $=100 \mathrm{kHz}$ are shown in Fig. 13c and Fig. 13d, respectively, where an IRN of $\sim 12.2 \mu \mathrm{V}_{\mathrm{rms}}$ is found in its $3-\mathrm{dB} \mathrm{BW}$ from $10 \mathrm{~Hz}$ to $25 \mathrm{kHz}$. For these measurements, an external reference current, $I_{\text {ref }}$, of $1 \mathrm{nA}$ and a sampling clock of $50 \mathrm{kHz}$ for the ADC are used. The small low-frequency peaking in the response is due to the mismatch between the pseudo resistors in the feedback network of the BBAMP, and can be reproduced in post-layout simulations. Under these conditions, a total power consumption of $0.56 \mu \mathrm{W}$ when using a single supply of $0.6 \mathrm{~V}$ is measured, including the power of on-chip buffers needed to distribute all clocks, and excluding the overhead for generating the on-chip reference current.

2) One-Way FMCW Ranging Measurements: A separate setup to characterize the FMCW ranging link is built in air at room temperature. The TX sends a Chirp via a PZT2, which is received by an identical PZT2, passed to the MAINRX and there mixed with an identical Chirp. The moment at which both Chirp signals begin is synchronized by a common start signal. The ADC data of the MAINRX are recorded during one sweep time, $T_{\text {sweep }}$ of $10 \mathrm{~ms}$. Afterward, the FFT of the recorded data is calculated in MATLAB. The FFT resolution of the derived spectrum is equal to the inverse of the recorded time $\left(T_{\text {sweep }}\right)$, which corresponds to $100 \mathrm{~Hz}\left(\frac{1}{10 \mathrm{~ms}}\right)$. The characterization of this FMCW ranging link up to $180 \mathrm{~cm}$ in air is shown in Fig. 17a and the absolute error between the calculated distance and the reference distance is depicted in Fig. 17b. The mean of the error in ranging is calculated as $0.18 \mathrm{~mm}$ with a standard deviation of $8.4 \mathrm{~mm}$. Modifying the equation to determine the distance $\mathrm{R}$ between nodes (Fig. 2) for a one-way trip of the US waves, a minimum measurable distance $d_{\min }=\frac{f_{\text {beat }} \cdot c_{U S} \cdot T_{\text {sweep }}}{B W}=26 \mathrm{~mm}$ is obtained from the minimum FFT resolution of $100 \mathrm{~Hz}$. Thus, as shown in Fig. 17b, the absolute error of this measurement will follow the trend of a quantization error of amplitude $d_{\text {min }}$, to which would correspond a theoretical standard deviation of $\sigma_{F F T 1}=$ $\frac{d_{\min }}{\sqrt{12}}=7.5 \mathrm{~mm}$. The FFT quantization is thus the main limiting factor to the ranging resolution in this experiment. To keep the post-processing as simple as possible, $f_{\text {beat }}$ (Fig. 2) is simply chosen as the frequency with the highest power signal

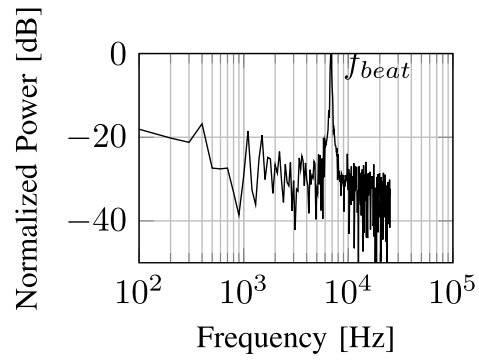

Fig. 18. A spectrum of the recorded ADC data at $1.8 \mathrm{~m}$ distance.

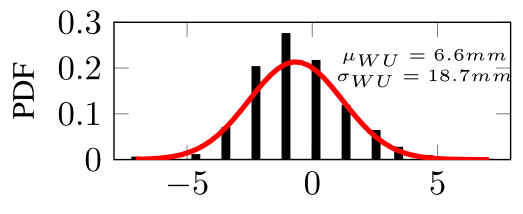

Absolute Error [cm]

Fig. 19. A normalized histogram of the 10000 measurements at $0.55 \mathrm{~m}$.

in the measured spectrum, as shown in Fig. 18. According to this characterization, the achievable distance in the FMCW ranging link can be up to $1.8 \mathrm{~m}$, where the $f_{\text {beat }}$ is successfully measured with at least $10 \mathrm{~dB}$ margin above the max. measured noise level.

\section{Rangefinder System Characterization}

A proof-of-concept characterization of the full proposed system, including the wake-up and ranging links is demonstrated here. The setup is the one shown in Fig. 1b, and described in Section II-A. Similarly to the one-way FMCW ranging measurements, a $50 \mathrm{kHz}$ sampling clock for the ADC is used and the ADC data of the MAINRX is recorded during a $10 \mathrm{~ms}$ of sweep time. The experiment is performed in air at room temperature without external synchronization. The LO of $38 \mathrm{kHz}$ from the on-chip clock generator is used in the WURX. To quantify the overall ranging error, NODE1 and NODE2 are placed at $0.55 \mathrm{~m}$ apart and the distance measurement of the full rangefinder system is repeated 10000 times. Fig. 19 shows the histogram of the absolute error, which is fitted to a normal distribution with a mean $\left(\mu_{W U}\right)$ of $6.6 \mathrm{~mm}$ and a standard deviation $\left(\sigma_{W U}\right)$ of $18.7 \mathrm{~mm}$. In this experiment, the jitter of the LO in the WURX link and the WURX ADC sampling result in an uncertainty of the wake-up time of the node, which reflects in an error in the distance measurement and in the observed standard deviation. In Fig. 20a, the rangefinder system measurements of the $f_{\text {beat }}$ up to $1 \mathrm{~m}$ are shown, where at each point an average of $\mathrm{N}=15$ measurements is reported. The averaging results in a decrease of the standard deviation due to random sources of uncertainty in the WURX. The deterministic error caused by the FFT resolution for the two-way ranging can be estimated using the $\mathrm{R}$ equation in Fig. 2, to obtain a minimum measurable distance $R_{\min }=$ $13 \mathrm{~mm}$. Changing the measurement distance will thus cause a uniformly distributed ranging error with a standard deviation $\sigma_{F F T 2}=\frac{R_{\min }}{\sqrt{(12)}}=3.7 \mathrm{~mm}$. Combining the two uncorrelated error sources, one would predict for the full system measurements a standard deviation $\sigma_{t o t}$, which can be estimated as $\sigma_{\text {tot }}=\sqrt{\frac{\sigma_{W U}^{2}}{N}+\sigma_{F F T 2}^{2}}=6.1 \mathrm{~mm}$. The measured error 


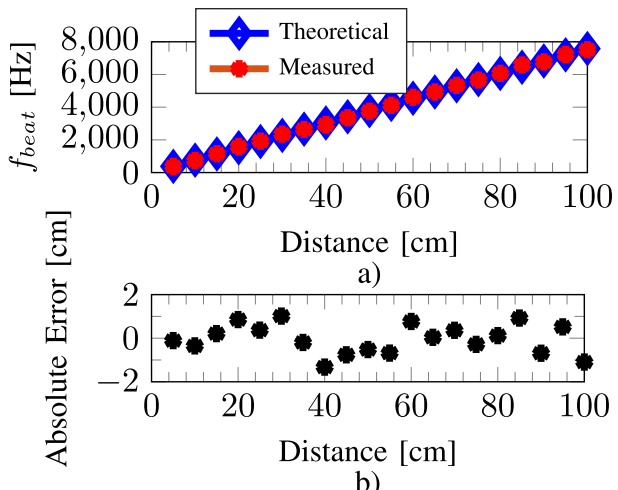

b)

Fig. 20. a) Full-system ranging link characterization. b) Absolute error of the rangefinder system. Each point is an average of 15 independent measurements.

TABLE III

PERformance Comparison of THE PRoposed US WuRX

\begin{tabular}{|c|c|c|c|c||c|}
\hline & {$[43]$} & {$[44]$} & {$[45]$} & {$[46]$} & This Work \\
\hline Technology [nm] & 65 & 250 & 65 & 65 & 65 \\
\hline Carrier Frequency [kHz] & 40 & 41 & 57 & 40 & 39 \\
\hline Supply Voltage [V] & 0.6 & 0.3 & 0.5 & 0.8 & 0.6 \\
\hline Modulation & OOK & OOK & OOK & OOK & OOK \\
\hline Data Rate $[\mathrm{bps}]$ & 250 & 250 & 336 & 1000 & 333 \\
\hline Receiver Power $[\mu \mathrm{W}]$ & 4.4 & 1 & 0.008 & 1.18 & 0.0236 \\
\hline Sensitivity $[\mathrm{dBm}]$ & -81 & -81 & -59.7 & -81.6 & -69.5 \\
\hline$F O M_{W u R x}\left[(J / b) \cdot W \cdot 10^{-20}\right]$ & 14 & 3.18 & 2.55 & 0.82 & 0.79 \\
\hline
\end{tabular}

in Fig. 20b has a mean of $0.5 \mathrm{~mm}$ and a standard deviation of $7 \mathrm{~mm}$, which is close to the expected value. It can be observed that the addition of the wake-up uncertainty randomizes the shape of the error in Fig. 20b compared to the sawtooth behavior observed in Fig. 17b.

\section{BENCHMARK}

The performance of the proposed US WURX and its comparison with recent prior-art is summarized in Table III, where a Figure-of-Merit for wake-up receivers proposed in [49] as $F o M_{W u R x}=\operatorname{Energy}(J) /$ bit $\cdot P_{\text {sensitivity }}(W)$ is used for benchmarking. The works in [43], [44], [46] achieve a sensitivity level of around $-81 \mathrm{dBm}$, while consuming more than $1 \mu \mathrm{W}$. In [45], the lowest power consumption of $8 \mathrm{nW}$ is reported with an envelope-detector (ED) first topology, where a lower sensitivity of $-59.7 \mathrm{dBm}$ is obtained. In this work, by using a mixer-first front-end, the low-conversion gain of the ED is avoided and the sensitivity of the WURX is improved. One should notice that the FoM $M_{W u R x}$ reported in [46] does not include, similarly to the present work, the negligible power overhead of the bias current generation. With a total of $23.6 \mathrm{nW}$ power consumption, our work achieves a $F O M_{W u R x}$ of $0.79(\mathrm{~J} / \mathrm{b}) \cdot \mathrm{W} \cdot 10^{-20}$, which in line with the best prior-art.

The performance of the proposed US rangefinder and its comparison with prior-art is summarized in Table IV. The works in [47], [48] are based on the processing of the echoes coming from passive reflectors in air. On the other hand, the rangefinder in [7] and this work enable US distance measurement between active sensor nodes. This work and [7] exploit a frequency division duplexing between their transmitters and receivers, thereby, the interference from passive reflectors is avoided, as demanded in our application. Compared to
TABLE IV

PERFormance Comparison OF THE Proposed US RANGEFINDER

\begin{tabular}{|c|c|c|c|c|c|c|}
\hline & [47] & [48] & \multicolumn{2}{|c|}{ [7] } & \multicolumn{2}{|c|}{ This Work } \\
\hline Method & ToF & Phase-shift & \multicolumn{2}{|c|}{ FMCW } & \multicolumn{2}{|c|}{ FMCW } \\
\hline Target Reflector & Passive & Passive & \multicolumn{2}{|c|}{ Active } & \multicolumn{2}{|c|}{ Active } \\
\hline Medium & Air & Air & \multicolumn{2}{|c|}{ Air } & \multicolumn{2}{|c|}{ Air } \\
\hline Transducers & PMUT & Membrane & \multirow{2}{*}{\multicolumn{2}{|c|}{ PZT }} & \multicolumn{2}{|c|}{ PZT } \\
\hline Technology $[\mathrm{nm}]$ & 180 & 800 & & & \multicolumn{2}{|c|}{65} \\
\hline Min/Max Range $[\mathrm{mm} / \mathrm{m}]$ & $45 / 1$ & $18 / 0.11$ & \multicolumn{2}{|c|}{$50 / 1$} & \multicolumn{2}{|c|}{$50 / 1$} \\
\hline Range Error $[\mathrm{mm}]$ & $0.41 @ 0.5 \mathrm{~m}$ & $2.5 @ 0.1 \mathrm{~m}$ & \multicolumn{2}{|c|}{$6.5 @ 0.55 \mathrm{~m}$} & \multicolumn{2}{|c|}{$18.7 @ 0.55 \mathrm{~m}$} \\
\hline Field of view & 3D & 1D & \multicolumn{2}{|c|}{ 1D } & \multicolumn{2}{|c|}{ 1D } \\
\hline TX Supply [V] & 32 & 5 & \multicolumn{2}{|c|}{$0.6+1.2$} & \multicolumn{2}{|c|}{$0.6+1.2$} \\
\hline & & & FMCW & FMCW & Wake-Up & FMCW \\
\hline Frequency $[\mathrm{kHz}]$ & 220 & 100 & $98-106$ & 194-202 & 39 & $94-107$ \\
\hline TX Energy Consumption $[\mu J]$ & 1.05 & 2800 & 1.07 & 1.59 & 0.43 & 0.82 \\
\hline RX Supply [V] & 1.8 & 5 & 0.6 & 0.6 & 0.6 & 0.6 \\
\hline RX Energy Consumption [nJ] & 1600 & $3.7 \cdot 10^{6}$ & 4.48 & 4.48 & 0.236 & 5.6 \\
\hline Always-on Power Consumption [nW] & N/A & N/A & \multicolumn{2}{|c|}{560} & \multicolumn{2}{|c|}{23.6} \\
\hline Total Energy per Measurement $[\mu J]$ & $2.65^{a}$ & 6500 & \multicolumn{2}{|c|}{2.67} & \multicolumn{2}{|c|}{1.25} \\
\hline
\end{tabular}

Energy consumption is scaled per channel excluding the power overhead of the digital circuits.

[7], this work uses a multi-level driver to improve the driving efficiency of the US transducers, which allows lowering the TX power consumption. In addition, this work uses identical Chirps residing in the same frequency band, which are digitally synthesized separately on the initiator and wake-up node without any analog or digital modification, thus simplifies the hardware needed in the nodes. Another profound difference of this work compared to [7], [48] and [47] is the use of a mixer-first WURX combined with FMCW ranging to lower the always-on power consumption of the nodes down to $23.6 \mathrm{nW}$. Although this work has a lower ranging resolution of $18.7 \mathrm{~mm}$, which is tolerable in our applications, the proposed rangefinder system dissipates $1.25 \mu \mathrm{J}$ per measurement, an improvement of at least $2 \mathrm{x}$ compared to prior art US rangefinders. This figure includes the power of the on-chip buffers used to distribute and drive the Chirp signals and the sampling clock of the ADC, while it excludes the power needed for generation of the on-chip current references and the clocks to synthesize the Chirp signals, the losses due to the generation of the $0.6 \mathrm{~V}$ and $1.2 \mathrm{~V}$ supplies from a battery, the memory leakage and the energy needed to write (read) the data to (from) an on-chip memory. An additional highlight of this work is that the response of the nodes in wake-up mode will be transmitted only when they receive a signal in a separate frequency band, thus their operation is independent of each other. This means that a global synchronization is not needed in the swarm of sensor nodes. Furthermore, the signal processing (e.g. FFT) needed to derive the spectrum of the received data and calculating the distances can be performed outside the environment under test, after the nodes are recuperated at the end of the exploration. As a result, the power overhead for digital processing can be minimized in the actual application. The memory needed to store one measurement is $10 \mathrm{~ms} \cdot 50 \mathrm{kS} / \mathrm{s}$. $10 b=5 \mathrm{~kb}$. The on-chip memory needed to store these data is not implemented yet. A recent work in [50] demonstrates a $1 \mathrm{Mb}$ flash memory which features a sleep mode power that is lower than the power consumption of the WURX. Based on the parameters provided by this work, the energy that would be spent to write and read the data needed per distance measurement, including the memory used to synthesize the Chirp, is only $20 \%$ of the total energy consumption per measurement. Furthermore, using ultra low-power approaches such as in [51], the memory leakage can be reduced even 
more, making it completely negligible when compared to the always-on node power achieved in this work.

\section{CONCLUSION}

This work presents the circuit and system design for an ultrasonic rangefinder system, where the sensor nodes can be operated in air or a fluidic environment. The nodes in a swarm perform measurements to find their relative distance while using separate frequency bands for the wake-up and FMCW ranging links. This makes the distance measurement insensitive to the echoes generated by the boundary of the environment being explored. The experimental characterization of the building blocks of the proposed system has been presented. The measurement results show that the proposed approach enables an always-on power consumption of only $23.6 \mathrm{nW}$ per node and makes possible ultrasonic distance measurements with the lowest energy per measurement reported to date.

\section{REFERENCES}

[1] J. Jang and F. Adib, "Underwater backscatter networking," in Proc. ACM Special Interest Group Data Commun., Aug. 2019, pp. 187-199.

[2] I. F. Akyildiz, D. Pompili, and T. Melodia, "Underwater acoustic sensor networks: Research challenges," Ad Hoc Netw., vol. 3, no. 3, pp. 257-279, May 2005.

[3] H. A. Duisterwinkel, "Exploring enclosed environments with floating sensors: Mapping using ultrasound," Ph.D. dissertation, Dept. Elect. Eng., Technische Univ. Eindhoven, Eindhoven, The Netherlands, Nov. 2019. [Online]. Available: https://research.tue.nl/en/publications/ exploring-enclosed-environments-with-floating-sensors-mapping-usi

[4] M. Andraud et al., "Exploring the unknown through successive generations of low power and low resource versatile agents," in Proc. Design, Autom. Test Eur. Conf. Exhib. (DATE), Mar. 2017, pp. 290-293.

[5] G. Berkol, P. G. M. Baltus, P. J. A. Harpe, and E. Cantatore, "Design of a low-power ultrasound transceiver for underwater sensor networks," in Proc. 14th Conf. Res. Microelectron. Electron. (PRIME), Jul. 2018, pp. $129-132$.

[6] E. Talnishnikh, J. van Pol, and H. J. Wörtche, "Micro motes: A highly penetrating probe for inaccessible environments," in Intelligent Environmental Sensing. Cham, Switzerland: Springer, 2015, pp. 33-49.

[7] G. Berkol, P. G. M. Baltus, P. J. A. Harpe, and E. Cantatore, "A 2.67 $\mu \mathrm{J}$ per measurement $\mathrm{FMCW}$ ultrasound rangefinder system for the exploration of enclosed environments," IEEE Solid-State Circuits Lett., vol. 3, pp. 326-329, 2020.

[8] X. Che, I. Wells, G. Dickers, P. Kear, and X. Gong, "Re-evaluation of RF electromagnetic communication in underwater sensor networks," IEEE Commun. Mag., vol. 48, no. 12, pp. 143-151, Dec. 2010.

[9] E. H. A. Duisterwinkel, G. Dubbelman, E. Talnishnikh, J. J. W. M. Bergmans, H. J. Wortche, and J.-P.-M. G. Linnartz, "Gowith-the-flow swarm sensing in inaccessible viscous media," IEEE Sensors J., vol. 20, no. 8, pp. 4442-4452, Apr. 2020.

[10] S. Schlupkothen, A. Hallawa, and G. Ascheid, "Evolutionary algorithm optimized centralized offline localization and mapping," in Proc. Int. Conf. Comput., Netw. Commun. (ICNC), Mar. 2018, pp. 625-631.

[11] M. G. Hinchey, J. L. Rash, W. F. Truszkowski, C. A. Rouff, and R. Sterritt, "Autonomous and autonomic swarms," in Proc. Int. Conf. Softw. Eng. Res. Pract. (SERP), Las Vegas, NV, USA, 2005. [Online]. Available: https://ntrs.nasa.gov/citations/20050210015

[12] H.-P. Tan, R. Diamant, W. K. G. Seah, and M. Waldmeyer, "A survey of techniques and challenges in underwater localization," Ocean Eng., vol. 38, nos. 14-15, pp. 1663-1676, Oct. 2011.

[13] M. Erol-Kantarci, H. Mouftah, and S. Oktug, "Localization techniques for underwater acoustic sensor networks," IEEE Commun. Mag., vol. 48, no. 12, pp. 152-158, Dec. 2010.

[14] R. M. Eustice, H. Singh, and L. L. Whitcomb, "Synchronous-clock, oneway-travel-time acoustic navigation for underwater vehicles," J. Field Robot., vol. 28, no. 1, pp. 121-136, Jan. 2011.

[15] J. Liu, Z. Wang, J.-H. Cui, S. Zhou, and B. Yang, "A joint time synchronization and localization design for mobile underwater sensor networks," IEEE Trans. Mobile Comput., vol. 15, no. 3, pp. 530-543, Mar. 2016.
[16] H. Attarzadeh, Y. Xu, and T. Ytterdal, "A low-power high-dynamicrange receiver system for in-probe 3-D ultrasonic imaging," IEEE Trans. Biomed. Circuits Syst., vol. 11, no. 5, pp. 1053-1064, Oct. 2017.

[17] J. Lim, C. Tekes, F. L. Degertekin, and M. Ghovanloo, "Towards a reduced-wire interface for CMUT-based intravascular ultrasound imaging systems," IEEE Trans. Biomed. Circuits Syst., vol. 11, no. 2, pp. 400-410, Apr. 2017.

[18] P. Behnamfar, R. Molavi, and S. Mirabbasi, "Transceiver design for CMUT-based super-resolution ultrasound imaging," IEEE Trans. Biomed. Circuits Syst., vol. 10, no. 2, pp. 383-393, Apr. 2016.

[19] G. Kokossalakis, "Acoustic data communication system for in-pipe wireless sensor networks," Ph.D. dissertation, Massachusetts Inst. Technol., Cambridge, MA, USA, 2006.

[20] Selection and Specification of Crystals for Texas Instruments USB 2.0 Devices, Appl. Note SLLA122, Texas-Instrum., Dallas, TX, USA, 2002.

[21] S. Roehr, P. Gulden, and M. Vossiek, "Precise distance and velocity measurement for real time locating in multipath environments using a frequency-modulated continuous-wave secondary radar approach," IEEE Trans. Microw. Theory Techn., vol. 56, no. 10, pp. 2329-2339, Oct. 2008.

[22] P. Barrenechea, F. Elferink, and J. Janssen, "FMCW radar with broadband communication capability," in Proc. Eur. Radar Conf., Oct. 2007, pp. 130-133.

[23] F. Lampel, R. F. Tigrek, A. Alvarado, and F. M. Willems, "A performance enhancement technique for a joint FMCW RadCom system," in Proc. 16th Eur. Radar Conf. (EuRAD), 2019, pp. 169-172.

[24] H. Slabbekoorn, R. J. Dooling, and A. N. Popper, Effects of Anthropogenic Noise on Animals (Handbook of Auditory Research). New York, NY, USA: Springer, 2018, pp. 119-120.

[25] H.-Y. Tang et al., "Miniaturizing ultrasonic system for portable health care and fitness," IEEE Trans. Biomed. Circuits Syst., vol. 9, no. 6, pp. 767-776, Dec. 2015.

[26] K.-J. Choi, H. G. Yeo, H. Choi, and D.-W. Jee, "A 28.7V modular supply multiplying pulser with $75.4 \%$ power reduction relative to $\mathrm{CV}^{2} f$," IEEE Trans. Circuits Syst. II, Exp. Briefs, early access, Aug. 27, 2020, doi: 10.1109/TCSII.2020.3019811

[27] G. Jung et al., "A reduced-wire ICE catheter ASIC with Tx beamforming and Rx time-division multiplexing," IEEE Trans. Biomed. Circuits Syst. vol. 12 , no. 6, pp. 1246-1255, Dec. 2018

[28] O. Farhanieh, A. Sahafi, R. B. Roy, A. S. Ergun, and A. Bozkurt, "Integrated HIFU drive system on a chip for CMUT-based catheter ablation system," IEEE Trans. Biomed. Circuits Syst., vol. 11, no. 3, pp. 534-546, Jun. 2017.

[29] MCUSR18A40B12RS-MCUSD40A100B17RS70C. (2020). Multicomp Ultrasonic Sensors. [Online]. Available: https://uk.farnell.com/ multicomp-ultrasonic-transducers

[30] M. I. Skolnik, RADAR Systems. New York, NY, USA: McGraw-Hill, 2001.

[31] S. Sendra, J. Lloret, J. M. Jimenez, and L. Parra, "Underwater acoustic modems," IEEE Sensors J., vol. 16, no. 11, pp. 4063-4071, Jun. 2016.

[32] C. Chen et al., "A front-end ASIC with receive sub-array beamforming integrated with a $32 \times 32$ PZT matrix transducer for 3-D transesophageal echocardiography," IEEE J. Solid-State Circuits, vol. 52, no. 4, pp. 994-1006, Jan. 2017.

[33] L. J. Svensson and J. G. Koller, "Driving a capacitive load without dissipating fCV $\mathrm{fC}^{2}$, in Proc. IEEE Symp. Low Power Electron., Oct. 1994, pp. $100-101$.

[34] K. Chen, H.-S. Lee, A. P. Chandrakasan, and C. G. Sodini, "Ultrasonic imaging transceiver design for CMUT: A three-level 30-Vpp pulseshaping pulser with improved efficiency and a noise-optimized receiver," IEEE J. Solid-State Circuits, vol. 48, no. 11, pp. 2734-2745, Nov. 2013.

[35] B. Razavi, RF Microelectronics (Prentice-Hall Communications Engineering and Emerging Technologies Series). Upper Saddle River, NJ, USA: Prentice-Hall, 2011.

[36] P. Harpe, H. Gao, R. V. Dommele, E. Cantatore, and A. H. M. van Roermund, "A $0.20 \mathrm{~mm}^{2} 3 \mathrm{nW}$ signal acquisition IC for miniature sensor nodes in $65 \mathrm{~nm}$ CMOS," IEEE J. Solid-State Circuits, vol. 51, no. 1, pp. 240-248, Jan. 2016.

[37] M. D. Seeman and S. R. Sanders, "Analysis and optimization of switched-capacitor DC-DC converters," IEEE Trans. Power Electron., vol. 23, no. 2, pp. 841-851, Mar. 2008.

[38] S. Jeong et al., "Always-on 12-nW acoustic sensing and object recognition microsystem for unattended ground sensor nodes," IEEE J. SolidState Circuits, vol. 53, no. 1, pp. 261-274, Jan. 2018. 
[39] N. Weste and D. Harris, CMOS VLSI Design: A Circuits and Systems Perspective. Boston, MA, USA: Pearson Education, Inc., 2010. [Online]. Available: http://pages.hmc.edu/harris/cmosvlsi/4e/index.html

[40] S. Robert, O. Abed-Meraim, and L. L. Coco, "Wide-band variable-gain LNA in $65 \mathrm{~nm}$ CMOS with inverter based amplifier for multi-tuners cable TV reception," in Proc. ESSCIRC, Sep. 2009, pp. 104-107.

[41] P. Wang and T. Ytterdal, "A 54- $\mu \mathrm{W}$ inverter-based low-noise singleended to differential VGA for second harmonic ultrasound probes in 65-nm CMOS," IEEE Trans. Circuits Syst. II, Exp. Briefs, vol. 63, no. 7, pp. 623-627, Jul. 2016.

[42] H. Wang and P. P. Mercier, "A 1.6\%/V $124.2 \mathrm{pW} 9.3 \mathrm{~Hz}$ relaxation oscillator featuring a $49.7 \mathrm{pW}$ voltage and current reference generator," in Proc. 43rd IEEE Eur. Solid State Circuits Conf. (ESSCIRC), Sep. 2017, pp. 99-102.

[43] K. Yadav, I. Kymissis, and P. R. Kinget, "A 4.4- $\mu \mathrm{W}$ wake-up receiver using ultrasound data," IEEE J. Solid-State Circuits, vol. 48, no. 3, pp. 649-660, Mar. 2013.

[44] H. Fuketa, S. O'uchi, and T. Matsukawa, "A $0.3-\mathrm{V} \quad 1-\mu \mathrm{W}$ superregenerative ultrasound wake-up receiver with power scalability," IEEE Trans. Circuits Syst. II, Exp. Briefs, vol. 64, no. 9, pp. 1027-1031, Sep. 2017.

[45] A. S. Rekhi and A. Arbabian, "Ultrasonic wake-up with precharged transducers," IEEE J. Solid-State Circuits, vol. 54, no. 5, pp. 1475-1486, May 2019.

[46] G. Berkol, P. G. M. Baltus, P. J. A. Harpe, and E. Cantatore, "A $-81.6 \mathrm{dBm}$ sensitivity ultrasound transceiver in $65 \mathrm{~nm}$ CMOS for symmetrical data-links," in Proc. IEEE 45th Eur. Solid State Circuits Conf. (ESSCIRC), Sep. 2019, pp. 145-148.

[47] R. J. Przybyla, H.-Y. Tang, A. Guedes, S. E. Shelton, D. A. Horsley, and B. E. Boser, "3D ultrasonic rangefinder on a chip," IEEE J. Solid-State Circuits, vol. 50, no. 1, pp. 320-334, Jan. 2015.

[48] C. Kuratli and Q. Huang, "A CMOS ultrasound range-finder microsystem," IEEE J. Solid-State Circuits, vol. 35, no. 12, pp. 2005-2017, Dec. 2000.

[49] S. Moazzeni, M. Sawan, and G. E. R. Cowan, "An ultra-low-power energy-efficient dual-mode wake-up receiver," IEEE Trans. Circuits Syst. I, Reg. Papers, vol. 62, no. 2, pp. 517-526, Feb. 2015.

[50] Q. Dong et al., "11.2 A 1Mb embedded NOR flash memory with $39 \mu \mathrm{W}$ program power for mm-scale high-temperature sensor nodes," in IEEE Int. Solid-State Circuits Conf. (ISSCC) Dig. Tech. Papers, Feb. 2017, pp. 198-199.

[51] S. C. Bartling et al., "An $8 \mathrm{MHz} 75 \mu \mathrm{A} / \mathrm{MHz}$ zero-leakage non-volatile logic-based Cortex-M0 MCU SoC exhibiting 100\% digital state retention at VDD $=0 \mathrm{~V}$ with $<400 \mathrm{~ns}$ wakeup and sleep transitions," in Proc. IEEE Int. Solid-State Circuits Conf. Dig. Tech. Papers, Feb. 2013, pp. $432-433$.

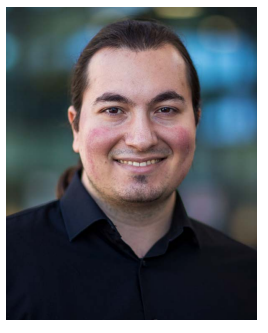

Gönenç Berkol (Member, IEEE) received the B.Sc. degree from Ege University, Izmir, Turkey, in 2013, and the M.Sc. degree engineering from Bogazici University, Istanbul, Turkey, in 2015, respectively, in electrical and electronics engineering. He is currently pursuing the Ph.D. degree in electrical engineering with the Eindhoven University of Technology, Eindhoven, The Netherlands. His research interests include low-power analog integrated-circuit design and design automation tools for analog circuits.

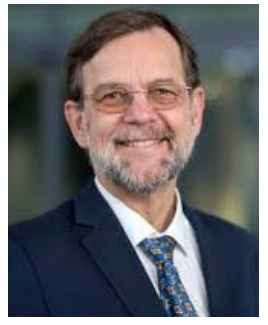

Peter G. M. Baltus (Senior Member, IEEE) was born in Sittard, The Netherlands, in 1960. $\mathrm{He}$ received the master's degree in electrical engineering and the Ph.D. degree from the Eindhoven University of Technology, Eindhoven, The Netherlands, in 1985 and 2004, respectively. He was a Research Scientist, the Program Manager, an Architect, the Domain Manager, the Group Leader, and a fellow with Philips, for 20 years, and later NXP, Eindhoven, The Netherlands, Nijmegen, The Netherlands, Tokyo, Japan, and Sunnyvale, CA, USA, where he focused on data converters, microcontroller architecture, digital design, software, and RF circuits and systems. In 2007, he joined the Eindhoven University of Technology, as a Professor of high-frequency electronics. From 2007 to 2016, he was the Director of the Centre for Wireless Technology, Eindhoven University of Technology, where he has been the Chair of the Integrated Circuits Group. He has coauthored more than 200 articles. He holds 16 U.S. patents.

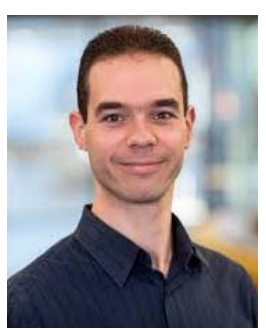

Pieter J. A. Harpe (Senior Member, IEEE) received the M.Sc. and Ph.D. degrees from the Eindhoven University of Technology, Eindhoven, The Netherlands, in 2004 and 2010, respectively. In 2008, he joined the Holst Center/imec, Eindhoven, as a Researcher. Since, he has been working on ultralow-power wireless transceivers, with a main focus on ADC research and design. In 2011, he joined the Eindhoven University of Technology, where he is currently an Associate Professor of low-power mixed-signal circuits. He was a recipient of the ISSCC 2015 Distinguished Technical Paper Award. He is a Co-Organizer of the yearly workshop on Advances in Analog Circuit Design (AACD) and an Analog Subcommittee Chair of the ESSCIRC Conference. He also served as a Member of the ISSCC ITPC and a Distinguished Lecturer of the IEEE SSCS.

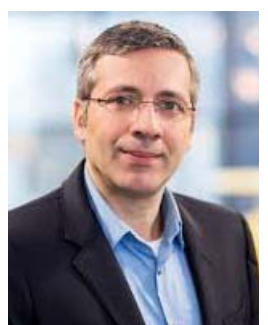

Eugenio Cantatore (Fellow, IEEE) received the master's and Ph.D. degrees in electrical engineering from the Politecnico di Bari, in 1993 and 1997, respectively. Till 1999, he was a fellow of the European Laboratory for Particle Physics (CERN), Geneva. In 1999, he moved to Philips Research, Eindhoven, and in 2007, joined the Eindhoven University of Technology, where he has been a Full Professor since 2016. He authored or coauthored more than 200 papers in journals and conference proceedings, and 13 patents. His research interests include the design and characterization of electronic circuits exploiting emerging technologies and the design of ultra-low power micro-systems. In 2006, he received the ISSCC Beatrice Winner Award for Editorial Excellence and was nominated in the Scientific American top 50 list. He received the Philips Research Invention Award in 2007, the Best Paper Award from ESSDERC 2012 and the Distinguished Technical Paper Award from ISSCC 2015. He has been active in the Technical Program Committees of ESSDERC and ISSCC and is a member of the Program Committees of IWASI and ESSCIRC. From 2013 till 2016, he has been chairing the ISSCC Technology Directions subcommittee, and in 2019, he was the ISSCC Program Chair. He is the Secretary of the Executive Committee of ISSCC and a member at large of the SSCS AdCom. He is an Associate Editor of the IEEE TRANSACTIONS on Circuit AND SyStems-I: Regular PAPERS. 